\title{
Understanding the Influence of Cultural Dimensions on the Interpretative Ability of People to Infer Personality from the Avatars: Evidence from Cultural Dimensions of Greece, Pakistan, Russia, and Singapore
}

\author{
Muhammad Nauman Shahid \\ National University of Singapore \\ nauman@,comp.nus.edu.sg
}

\author{
Maria Kralli \\ Saint Petersburg State University \\ marykralli@gmail.com
}

\begin{abstract}
Avatar is a customized cartoon representation of the self and many people develop inferences about individuals' online representations through their avatar's facial appearance. Research has shown that avatars can signal information about the personality and social desires of a person [1]. Nonetheless, customizing an avatar enables control of selfrepresentation that could potentially moderate the true personality traits of an individual. The customized facial appearance of the avatar affects people's ability to draw expressions [2], whereas, several cultural dimensions affect the interpretative ability of the people to construct personality inferences from the facial appearance of avatars. We found a significant relationship between neuroticism to uncertainty avoidance and masculinity, whereas, negative relationships were found between extraversion and masculinity, and agreeableness to uncertainty avoidance. The study uses three-dimensional avatars to capture detailed features and expressions on avatar faces.
\end{abstract}

\section{Introduction}

Modern technological advances have created virtual spaces where people gather and interact with each other (e.g. chat rooms, gaming) [3]. In some places, individuals represent themselves with a graphical image known as an avatar. Typically, the avatar is a representative image of the self in the virtual world that ranges from simple drawings (e.g. Nintendo characters known as MII) to the quite detailed threedimensional rendering of a character (e.g. World of Warcraft). There is a growing body of research that has investigated the potential social outcomes of using avatars in virtual spaces [4]. In particular, research has demonstrated that avatars can signal information about the personality and social desires of a person such as friendship from others [1]. Avatars enable individuals to suppress or express various psychological and physical traits $[5,6,7]$. This ability to represent selfindicates the importance of creating personality inferences about avatar creators from the facial features of avatars. Although avatars can signal personality information of the avatar creator, perceiving personality inference is highly dependent on the cultural dimensions. Customization also enables control of self-representation that can moderate the true personality traits of an individual. This customization can further moderate the accuracy with which an individual is perceived or the social consequences of judgments based on the avatars. Therefore, this research attempts to understand the effect of cultural dimensions on creating personality inferences about avatar creators from their representative avatars. The facial features of the avatars include other characteristics such as the selection of costume, body type, and race etc. We also investigate both the accuracy of forming personality perceptions within the world of avatars, and true personality perceptions formed from actual human faces, and the social consequences of those inferences. The paper is organized as follows: Section 2 discusses the related work on avatar expressions and develops an understanding from the perspective of computermediated communication. Section 3 conceptualizes cultural dimensions from Hofstede's [8] work. Section 4 discusses the Brunswik lens model. Section 5 provides an overview of the methodology adopted for this study, mechanizes confirmatory analysis to understand the relationship between cultural dimensions and developing inference of big five personality traits from avatars.

\section{Related work}

\subsection{Construction of impressions in computer- mediated communication (CMC)}

Forming impressions is a critical aspect of any social interaction in routine life. Computer-Mediated Communication (CMC) affects the mechanism of forming impressions by specific $\mathrm{CMC}$ cues. These 
cues can be nominal and text-based, such as usernames, emoticons, and even writing style [9, 10] but even these nominal cues can be used to form impressions of useful social categories such as gender and disposition $[9,11]$. In general, these CMC-specific cues reveal accurate information about individuals' personality, however, a high degree of accuracy is achieved in text-based interactions [12], despite the availability of simplest of the cues such as an email address [13]. However, some researchers show that this accuracy is lower than that found in face-to-face interactions [14].

Online social networking profiles $[15,16]$ and personal websites [17] comprise both verbal and nonverbal cues, and these have also been associated with accurate personality judgments.

\subsection{Impressions based on avatars}

People use avatars in online environments that differ in their implementation, from static images to dynamic three-dimensional characters $[1,18]$. Avatars can promote social relationships, increasing feelings of connectivity and emotional involvement [19]. People appear to use visual cues encoded in the avatar to form an impression of the avatar and its user. Individuals often anthropomorphize avatars [20], it is evident that impressions are perceived from avatars in a manner similar to how they form impressions of individuals in the real world [21]. For example, avatars that have tattoos are perceived as being sensationseeking and risk-taking [22]. The perceived attractiveness and credibility of a human is cued to androgynous and human alikeness of the avatar [23], and these evaluations are indirectly related to the credibility of the avatar's user [23]. In addition, these same cues impact whether users would like to be represented by a given avatar [23, 24]. Therefore, the visual characteristics of avatars play an important role to shape online perceptions about individuals who choose to represent themselves. However, whether cues accurately reflect and signal an individual's realworld traits?

There are several reasons to think that an avatar's cues may not accurately display the impressions of its user. First, the users in virtual environments might adopt identities that are different from their real-world identity that might be well suited for identity exploration [25]. Second, avatars can be easily customized, that means the user can easily control the appearance and can embody any wishful characteristics $[6,26]$. Lastly, avatars may provide an opportunity to the individual to reflect themselves differently than their actual social profile [7], motivated by entertainment, desires, or enjoyment to manage self-presentation $[7,26]$. The latter is achieved by emphasizing the desired psychological traits or physical characteristics, such as confidence, attractiveness, or intelligence $[6,26,27]$. The potential reason behind this assumption is that positive evaluations are often extended to its user [23], therefore, individuals may be highly convinced to customize avatars in ways that are highly inconsistent with reality, resulting in inaccurate impressions formed by others.

In contrast, there are reasons to believe that avatars may have valid identity cues. There might be individuals who are uncomfortable or marginalized in the real world and may have virtual worlds to express their true-selves [7]. Researchers have borne out the idea that individuals often choose avatars to customize and reflect their real personalities, mental conditions, and interests $[25,28]$. Individual characteristics such as self-esteem, gender, and personality, guide avatar customization [26]. For example, our choice of clothes or wearable accessories are representative features of ourselves and they convey certain information cues to others [29], likewise, the accessories or representative features we choose for our avatars may serve a similar function and may even correspond to our actual clothes [30]. Consistent with the clue that avatars can truthfully reflect identity, individuals prefer to choose avatars that are perceived similar to themselves [23, 31]. Additionally, there is a stream of research that supports the idea that individuals may be motivated to create and employ avatars that are representative of their true identity. In this study, we use the Brunswik Lens model to examine the accuracy of personality perceptions based on avatars [16].

\section{Cultural dimensions}

There is a growing body of research that examines the cultural aspects of virtual embodied agents. Ruttkay [32] discussed the design of facial expressions for virtual agents with a specific culture, and [33] modelled multimodal interactions with virtual agents by integrating culture as a computational parameter. However, only a few studies compared the cultural differences in interpreting avatars' facial expressions. For example, [2] found that cultural differences influence the interpretation of facial expressions differently across a range of diverse nationalities. Although, the findings showed a positive association between culture and interpretation of expressions, however, [2] conceptualization of culture was limited to the language and country of the participants. We believe that language is a constituent of many cultural dimensions that affect impression forming from avatars. [34] compared interpretations of animated gestures of avatars by Dutch and Japanese. They found cultural differences in the valences they perceived in animated characters. The Japanese subjects found strong feelings in bowing gesture of avatars than the 
Dutch subjects, even though there were no general differences in the interpretation of the presented gestures. [35] conducted a cross-cultural experiment where he observed a series of discussions between Chinese and Japanese subjects on a multilingual bulletin board system (BBS) that had expressive avatars designed by Japanese artists. The results showed that both Chinese and Japanese subjects interpreted some facial expressions completely differently. For example, the Japanese subjects interpreted "wide-eyed" expression as "surprised", while the Chinese subjects interpreted it as "intelligent"' and used it when presenting a novel idea or asking questions. The Japanese subjects tried to understand the meaning of the Chinese subject's message with the "wide-eyed" expression. This is one example of different cross-cultural interpretations of avatar expressions.

Cultures typically have two types of elements i.e., hidden and visible. Visible elements include symbols, artefacts, and practices; art and architecture; language, colour, and dress; social etiquette and traditions. However, visible cultural differences make only $10 \%$ of our cultural identities. Hidden cultural elements include values, assumptions, and beliefs that represent the remaining $90 \%$ of our cultural identity. The visible elements of culture particularly language has been considered substantially by existing researchers, however, in this study, we took a holistic view of culture to understand its effects on the evaluative ability of people to develop personality inferences about avatar creators.

To understand the relationship between personality and culture, we compare societies even if the data has partly been collected from individuals within those societies [36]. Because cultures are wholes and their internal logic cannot be understood in the terms used for the personality dynamics of individuals. Eco-logic differs from individual logic. Researchers have attempted to measure the culture dimensions in individuals. This has been tried most often in the case of individualism-collectivism, with notably inconsistent results [37]. Another way of doing it is to measure personality traits at the culture level by calculating the ratings of national character [34], which echoes shared perceptions of the personality traits of the typical member of the culture, or by expert ratings of the ethos itself described in the language of personality, as when [38] described Zuni culture as apollonian. Here, it is operationalized as the mean level of traits in individuals from the culture.

Geert Hofstede is a Dutch social psychologist, who has been influential in exploring national cultures. He investigated people in IBM in fifty different countries and developed six cultural dimensions that could distinguish one culture from the other. He defines culture as "the collective programming of the mind which distinguishes the members of one group or category of people from another" [8]. According to him, culture is learnt in the nurture and not inherited by human nature. The "collective programming of the mind" means that culture is a collective activity that is to be conceived as a dynamic process rather than a passive state. The other part of the definition "which distinguishes the members of one group or category of people from another" shows the individual and group identity formation and sustenance facets of enculturation in social institutions like family, school and work. Culture comes from the similarity of individuals within a cohort group (be it a linguistic community, an ethnic group or a scientific community) and in that sense it is collective. This similarity is not intended to be exact; neither does it imply essentialist homogeneity. In a multicultural society, culture is about collective particularity.

In this research, we operationalize culture as the mean level of traits in individuals of a society. According to Hofstede [8], the mean level of traits is referred from six cultural dimensions i.e., individualism versus collectivism, power distance, uncertainty avoidance, long-versus short-term orientation, masculinity versus femininity, and indulgence versus restraint. We conceptualize each of these six dimensions from [39] in subsequent sections.

\subsection{Individualism vs. collectivism}

Individualism is the opposite of collectivism that represents the degree to which individuals look after themselves or integrate themselves into groups [40]. Individualistic societies tend to have a self-focused view that only extends to his or her immediate family; whereas, collectivism-oriented societies have a people-group view from cradle to grave of lifetime protection in exchange for unquestioning loyalty.

\subsection{Power distance}

Power distance is defined as "the extent to which the less powerful members of institutions and organizations within a country expect and accept that power is distributed unequally" [40]. There are two elements of power distance i.e., high power distance, and low power distance.

In high power distance societies, hierarchical systems of assigned roles organize behaviour and power distance is defined from bottom up. The less powerful members expect and accept inequalities. Power is anticipated to provide relational harmony, role stability, and social order. Only a few people have access to resources, knowledge, and skills. Different social groups have differential involvement in governance. 
In low power distance societies, members believe that inequalities should be minimized. Power is seen as a source of corruption, coercion, and dominance. People recognize one another as moral equals with shared basic human interests. Members care about the welfare of others and cooperate with one another. Low power distance societies have large middle classes. They have transient and shareable power bases (e.g., knowledge, skill etc.). There are high upward social mobility and mass availability of resources and capabilities. Different social groups enjoy equal involvement in governance.

\subsection{Uncertainty avoidance}

Uncertainty avoidance is defined as "the extent to which members of a culture feel threatened by ambiguous or unknown situations" [40].

In weak uncertainty avoidance societies, members are comfortable with unclear and unknown situations. They are tolerant of change. Members hold multiple ideas as valid and accept different viewpoints. They are contemplative, emotionally stable, and relaxed. They prefer fewer rules and breaking rules is allowed. They prefer to rely on the word of others they trust rather than enter into contractual relationships. They are not concerned with orderliness and keeping written records.

In strong uncertainty avoidance societies, members are threatened by uncertainty, have an emotional need for predictability, and exhibit high resistance to change. This resistance is stated through nervousness, stress, and attempts to control the environment. Members formalize their interactions with others, verify communications in writing, and take relatively moderate and calculated risks. Members have rigid beliefs and strict behavioural norms, formal rules and law, and intolerance of rulebreaking or orthodoxic ideas or behaviours.

\subsection{Long-versus short-term orientation}

Time orientation, i.e. short term and long term, represents the extent to which members of a culture are cognitively programmed to accept delayed gratification of material, social, and emotional needs [40].

Cultures with a long-term orientation have a strong tendency and willingness to imagine future possibilities. Members set long-term goals, create plans, and work hard and persevere to achieve their ambitions. They delay gratification and display a strong propensity to save and invest. They are psychologically healthy and socially well-adjusted because they feel in control of their lives, but they may neglect current social relationships and obligations, and can fail to 'stop and smell the roses'.
Members of short-term orientated societies focus more on the present and past than in the future. They value instant satisfaction and prefer to spend now rather than save for the future. They live in the moment and are not concerned with past or future anxieties. They may engage themselves in risky, pleasureseeking pursuits and fail to recognize the negative longer-term implications of their indulgences.

\subsection{Masculinity vs femininity}

Masculinity embodies a society where emotional gender roles are clearly distinct: men are supposed to be assertive, tough, and focused on material success; women are supposed to be more modest, tender, and concerned with the quality of life [40]. Feministic societies overlap the gender roles - both men and women are expected to be modest, tender, and concerned with the quality of life. There are few women in positions of authority, a low percentage of women in the labour force, and occupational sex segregation. In these societies, females have lower levels of education and literacy relative to males. In addition, women hold a lower status in society and play a smaller role in community decision-making compared with men.

In summary, the masculinity side of this dimension is associated with preference in the society for accomplishment, heroism, insistence, and quantifiable rewards for achievement. Society at large is more competing. Whereas, femininity side stands for a preference for cooperation, humility, caring for the poor and weak and quality of life. Society at large is more agreement-driven.

\subsection{Indulgence vs. restraint}

Indulgence orientated societies embolden pleasure-seeking. The society allows relatively free gratification of basic and natural human drives to pursue fun activities for the sake of personal enjoyment. The societies are more extroverts and have strong perceptions for personal life control.

Whereas, restraint societies suppress gratification and hedonistic pleasures and regulate it by strict social norms. The societies are more introverts and exhibit "what happens to us is not our doing".

\section{The Brunswik lens model}

The Brunswik Lens Model suggests that observable cues found in the environment (e.g., cues present in customized avatars) provide a lens through which perceivers observe constructs that may not be directly observable [41]. Accuracy in a personality perception is driven by two components - 1) validity 
of the cue and 2) utilization of the cue. The validity of the cue refers to the relationship between phenomena (e.g., personality) and observable cues, whereas, utilization of the cue refers to the relationship between cues and how they are employed by perceivers. The accuracy occurs when there is a high degree of convergence between the cue validity and cue utilization.

In this study, we conceptualize good and bad sources of personality information across many types of stimuli using Brunswik Lens Model [41].

\section{This study}

The primary research focus of this study is to develop an understanding of interpreting five big personality inferences from the facial features of avatars and the influence of cultural dimensions on these interpretations. In the first step, we conceptualize interpretation of five big personality traits (also known as OCEAN) through Hofstede's [40] six cultural dimensions. The linear association between five-factor personality traits and six cultural dimensions are measured through the Pearson correlation analysis. The differences between gender and five-factor personality traits are determined through multivariate analysis. Also, multivariate analysis tests the differences between gender-and-five personality traits and gender-and-six cultural dimensions. Following the methodology of [36], the hypotheses are framed to test for linearity in the relationship between each of the five personality traits and each of the six cultural dimensions.

Table 1. Results of mean factor scores against the culture scores [36]

\begin{tabular}{|c|c|c|}
\hline Personality Trait & Dimension & $\begin{array}{l}\text { Cumulativ } \\
\text { e Adj. } \mathbf{R}^{2}\end{array}$ \\
\hline \multirow[b]{2}{*}{ Neuroticism } & +Uncertainty & 0.31 \\
\hline & $\begin{array}{l}+ \text { Masculinit } \\
\mathrm{y}\end{array}$ & 0.55 \\
\hline \multirow{2}{*}{ Extraversion } & $\begin{array}{l}\text { +Individuali } \\
\text { sm }\end{array}$ & 0.39 \\
\hline & -Masculinity & 0.46 \\
\hline \multirow{3}{*}{ Openness } & $\begin{array}{l}+ \text { Masculinit } \\
\mathrm{y}\end{array}$ & 0.13 \\
\hline & $\begin{array}{l}\text {-Power } \\
\text { distance }\end{array}$ & 0.29 \\
\hline & $\begin{array}{l}\text { +Uncertainty } \\
\text { avoidance }\end{array}$ & 0.36 \\
\hline Agreeableness & $\begin{array}{l}\text {-Uncertainty } \\
\text { avoidance }\end{array}$ & 0.28 \\
\hline Conscientiousness & $\begin{array}{l}\text { +Power } \\
\text { distance }\end{array}$ & 0.24 \\
\hline
\end{tabular}

\subsection{Theoretical Framework}

Figure 1 shows our theoretical model to determine personality inferences from avatars.

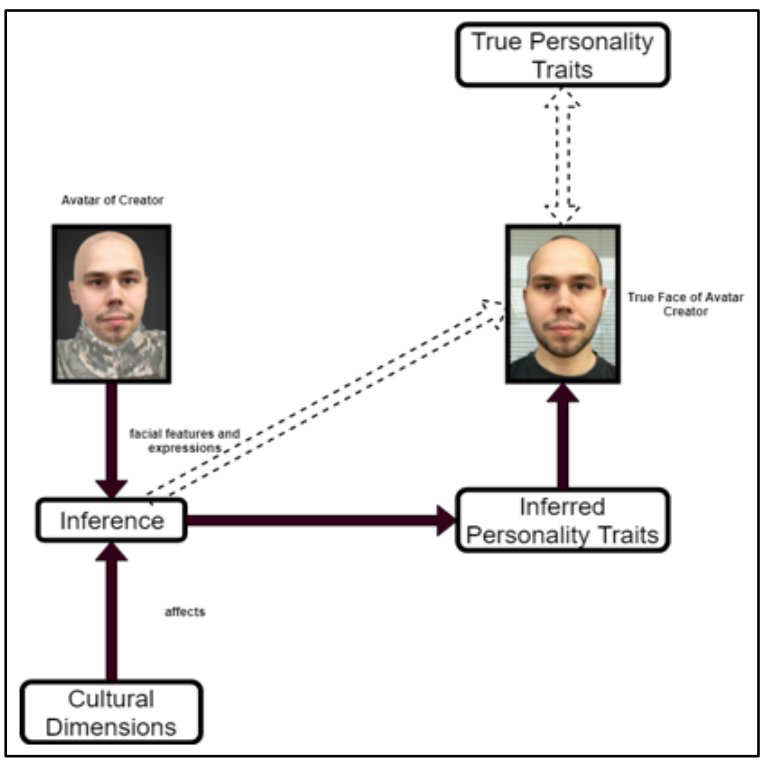

Figure 1. Theoretical model of personality inference from avatars

\subsection{Constructs operationalization}

We operationalize the cultural dimensions of each participant from [42] and determine the national score of the participant's country for each of six cultural dimensions. Whereas, to discuss the relationships between personality inferences and the influence of cultural dimensions, our pilot study consists of participants from four countries i.e., Greece, Pakistan, Russia, and Singapore. The countries have been chosen randomly for preliminary analysis. Figure 2 shows the indexes of cultural dimensions according to [42].

\section{Experiment}

\subsection{Method}

The study has two phases. In the first phase, the participants create customized avatars, and in the second phase, a different set of participants will view and rate the avatars created in the first phase.

\subsection{Participants}

The participants are not required to have a professional background in creating avatars. Instead, 
they will do it with a software tool designed for the experiment.

Each participant's demographic information such as age, gender, country, and each of the six indexes of national cultural dimensions will be recorded. We take an average of age across all participants and standard deviation between the ages and report the values.

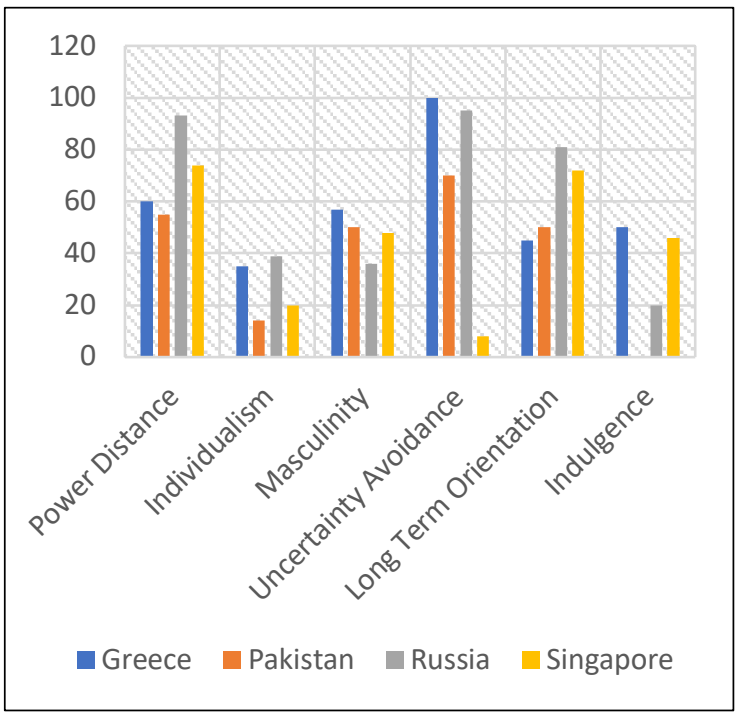

Figure 2. Cultural dimensions index of Greece, Pakistan, Russia, and Singapore [42]

\subsection{Materials}

Participants create avatars of themselves using the given software tool. The software tool allows them to choose a detailed 3D form of their avatar where they can customize many characteristics of body and accessories. For example, physical body type, it's colour, facial expressions, racial identification, clothing accessories etc. The consent of participants is taken to present their created avatars to other research participants.

\subsection{Big Five Inventory (BFI)}

The personality of the participants is assessed using the Big Five Inventory-44 (BFI-44) [43]. The BFI-44 is based on the five-factor model of personality and assesses the five major traits - a) openness, b) conscientiousness, c) extraversion, d) agreeableness, and e) neuroticism. This measure consists of forty-four descriptive phrases that are rated by the respondents with respect to self-characterization. The responses are given using a five-point Likert scale that ranges from 1 (strongly disagree) to 5 (strongly agree). For example, "I see myself as someone who is full of energy" represents extraversion, and "I see myself as someone who gets nervous easily" shows neuroticism.
The BFI- 44 is a valid and reliable method of measuring five-factor personality [44]. We use scores from the BFI-44 as a comprehensive measure of personality in all analyses that examined how creators' personalities might relate to being accurately perceived.

[45] presented the Big Five Inventory-10 (BFI-10) which is an abbreviated version of the BFI. BFI-10 measures the five-factor traits by two items, resulting in a total of 10 items. Each trait is measured by one true-scored item and one reverse-scored item. For example, extraversion is measured by the two items, "I see myself as someone who is outgoing, sociable" and "I see myself as someone who is reserved." Respondents rate each statement on a five-point Likert scale that ranges from 1 (strongly disagree) to 5 (strongly agree). Despite its briefness, the BFI-10 has proven good test-retest reliability, as well as good convergence with more detailed assessments of personality such as the 44-item BFI [45]. We use BFI10 only to determine profile level accuracy, to allow for a direct comparison between self-reported personality profiles and perceived personality profiles.

\subsection{Procedure}

There are two phases of this experiment. In the first phase, participants will create an avatar and subsequently complete the Big Five Inventory (BFI44). The participants are divided into two halves. The instruction to one half is: "create an avatar representation of yourself." Whereas, we give additional instruction to the second half: "your avatar should represent who you really are (e.g., your personality); remember, your avatar does not need to look alike you!". The avatar creators are required to complete the BFI-10 because this would be the measure that perceivers would later employ to infer personality from the avatar. The BFI-10 scores of the creators allow to make a direct comparison between self-rated personality and inferred personality.

Finally, we collect demographic information. The data for the second phase is collected online e.g. using an online survey portal such as Qualtrics. The second set of participants, with no overlap from the first phase, are shown a subset of 18 to 20 avatars created by participants in the first phase. The subsets are randomly assigned to the participants however, during the recruitment phase, the second phase participants are told that they will rate a subset. The standard instructions are: "you will be shown a series of 3dimensional avatars and you will rate each of them based on your perception about the personality of its creator". The cultural dimensions of this set of participants are assumed according to the scores reported in Figure 2. We also provide a questionnaire to these participants that list a number of 
characteristics that may or may not describe the individual they are rating. They examine each avatar and try to predict the personality of the person who created that avatar.

To evaluate consensus among raters (i.e., agreement on BFI personality ratings among all raters and across all avatars), we perform overall mean of single-perceiver interrater consensus. This consensus can be calculated using an intra-class correlation. The overall mean average-perceiver interrater consensus (i.e., agreement of personality ratings across raters within each subset) is also calculated.

The aforementioned exercise gives us a set of potential cues based on the avatar customization options, and the number of avatars possessing any given cue is noted. We employ independent coders to code all the cues for a given avatar. For all continuous cues (e.g., rated stylishness), coder ratings are averaged. The mean inter-judgement is calculated by correlating the two raters' scores on each continuous item and then averaging correlations across items. Inter-judge agreements across all items are also averaged. For binomial cues (e.g., brown hair), any disagreement between raters will be resolved. Cue utilization is calculated by correlating the coded physical cues of the created avatars with the average perceived score for each trait. Calculating cue validity followed a similar format but employed the avatar cues and self-reported personality traits from the BFI44.

\section{Preliminary results and discussion}

In this section, we organize our preliminary findings based on personality interpretations done by the participants and use logical reasoning to discuss results. The interpretation in our discussion is limited to participants who mentioned Greece, Pakistan, Russia, and Singapore as their national origin in demographic information.

The participants from Greece indicate negative correlations to agreeableness and a high score of 100 for uncertainty avoidance (UA) shows that Greeks are not comfortable in ambiguous situations [40] and while their personality perceptions about avatar creators are negatively correlated to agreeableness; openness and neuroticism are strongly correlated.

The index for individualism and masculinity in [42] is 35 and 57 for Greece that indicates a strong correlation to neuroticism and extraversion.

Pakistan shows a null score for indulgence vs. restraint (IVR) dimension which indicates a high restraint society and very regulated conduct of people's behaviour [40]. The interpretations of Pakistani participants show negative correlations between agreeableness and IVR however, interpretations for agreeableness trait are better than those of Greek participants. Whereas, lower values for masculinity (i.e., 50) and individualism (i.e., 14) dimension weakly correlates to extraversion and neuroticism for Pakistani participants.

Russia shows a high 81 score for long term orientation (LTO) dimension which means that Russian societies maintain their links to the past while dealing with present or future situations [40]. Therefore, the high uncertainty avoidance (UA) is positively correlated to LTO and that signals a weak interpretation for agreeableness. Interestingly, Russians show a peculiar correlation for masculinity (36) and individualism (39). On one hand, neuroticism is strongly correlated to masculinity dimension, however, on the other hand, masculinity is negatively associated with extraversion. Therefore, cultural logic weakly supports their interpretation of these two personality traits.

Unlike Greece which has highest uncertainty avoidance (UA) score of 100, Singapore has the least score of 8 for UA among all four countries. UA is positively correlated with neuroticism and openness while a negative relation exists with agreeableness. The individualism dimension is slightly better than Pakistan and shows better correlation for extraversion.

Greece and Russia are located in Europe and there are several regional and cultural similarities. The uncertainty avoidance (UA) and individualism for both countries are the highest among all four countries. The number of interpretations for extraversion was slightly higher by a factor of 0.3 for Russian participants than Greek participants.

Pakistan and Singapore are located in Asia and enjoy the continental neighbourhood. Despite greater economic differences, the participants of these countries show similar trends for major dimensions. However, uncertainty avoidance and indulgence are strongly the opposite. Interestingly, for Singaporeans, cultural logic does not clearly interpret openness and agreeableness.

The proposed assumption that cultural dimensions affect personality inferences is supported in our analysis. In the subsequent sections, we offer methodological evidence to further validate our claims.

\subsection{Evidence of inferring individual personality traits accurately from avatar cues}

We calculate the accuracy of traits by correlating the average rating of each trait with centred selfreported creator scores on the Big Five Inventory (BFI-44). Because the subsets of avatars will be rated by subsets of perceivers, a multilevel approach is used. The fixed effect from the model is standardized and represents the average relationship between the 
creator's self-report and perceiver's ratings of that trait, on average across perceivers.

We believe that this analysis postulates accurate information regarding trait extraversion, agreeableness, and neuroticism but not conscientiousness or openness. The relationship between cue utilization and cue validity provides insights for the accuracy of traits. Therefore, vectorcolumn correlations give insight into whether cue choices associated with creator personality were also utilized by perceivers [46]. The correlations of cue utilization and cue validity are transformed using Fisher's R-to-Z formula form vectors and then these vectors are correlated across all cues for each of the five big personality traits.

The vector correlations for extraversion, agreeableness, and neuroticism are positively significant, whereas, less significance is observed for conscientiousness and openness. This shows that individuals customize avatars to reflect their own traits in a way congruent to how perceivers use those avatar cues to infer personality based on cultural dimensions.

The concern that whether the valid and utilized cues can explain the accurate perception of each trait can be mitigated by conducting a series of bootstrapped multiple mediation analysis. For each personality trait, we conduct a regression with creator personality predicting rated personality traits and entered avatar cues that are both utilized and valid as potential mediators. We expect a positive mediation in terms of the non-zero total indirect effect of the cues for extraversion (e.g., shorts and jewellery) and agreeableness (e.g., open eyes and a neutral expression). The total indirect effect of the cues for neuroticism (grey or light brown shoes) and openness (number of accessories) would approach significance if the lower bound of the bootstrapped $95 \%$ confidence interval (CI) includes zero. That means cues for conscientiousness are valid and utilized.

\subsection{Evidence of inferring personality profiles accurately from avatar cues in light of cultural dimensions}

To examine the accuracy of personality inference from avatars, we consider cultural dimensions. The association of personality to dimensions adapted from [36] serves as a good starting point to explain our assumptions. Table 1 shows a comparison of cultural dimensions to personality traits and our analysis interprets values of the accuracy of profile interpretation from BFI-10 responses that correlate with the mean BFI-10 profile provided by the perceivers to directly compare perceived and selfreported personality. We assume raw associations to exist as a measure of overall accuracy and we perform single sample t-test with the null-hypothesis being "no correlation exists between self-reported creator personality and rated personality". The test value will be null for our assumption to be true.

\subsection{Evidence of personality traits that predict the perceived accuracy of actual personality traits of avatar creator}

To identify which personality traits of avatarcreator-are-associated with creating-an-avatarperceived accurately, we can use self-reported traits of personality of the creators in our multilevel model. We believe that individuals with extraversion, agreeableness, and conscientious traits are highly likely to be perceived with distinctive accuracy, whereas individuals who are more neurotic are less likely to be perceived with distinctive accuracy. The openness of the creator may not relate to being perceived with distinctive accuracy. Therefore, we include all five traits simultaneously as potential moderators to examine which of them uniquely predict accuracy. The values for gender and age included as covariates. The creator's extraversion, agreeableness, and neuroticism is a unique predictor, with extraversion and agreeableness predicting greater distinctive accuracy and neuroticism predicting less accuracy.

\section{Conclusion}

Rapid technological advancements have led people to infer information from virtual online spaces. Avatars are one such representation of individuals in the modern world that gives an interesting avenue to understand the underlining cues of personality interpretation. Although, it is a timely and relevant undertaking especially given the rapid explosion of online interactions in recent years. We suggest that inferring personality traits online can be more informative than meeting that individual in person because of the wealth of information provided by personal web pages and/or social networking profiles. The findings of this study give strong evidence that personality information can accurately be inferred from an individual's avatar.

\section{Future Work}

In the future, the study will be expanded to include participants from many different countries and regions. We predict interesting patterns given the variety of individuals with multiple nationalities. In particular, it would be interesting to know, is there a change in an individual's perceptions when the person is exposed to a new culture different than origin culture and how does the length of exposure to a 
different culture changes interplay with this likelihood. Additionally, it would be interesting to investigate how personality perception influences the perceiver's behaviour and interaction with that avatar. For example, if a person is more hostile to that person or more likely to harass them based on certain perceived personality traits, and how does this likelihood differ by culture or country.

\section{References}

[1] J. F. Bélisle and H. O. Bodur, "Avatars as information: Perception of consumers based on their avatars in virtual worlds," Psychol. Mark., vol. 27, no. 8, pp. 741-765, Aug. 2010.

[2] T. Koda, T. Ishida, M. Rehm, and E. André, "Avatar culture: Cross-cultural evaluations of avatar facial expressions," in AI and Society, 2009, vol. 24, no. 3, pp. 237-250.

[3] C. A. Steinkuehler and D. Williams, "Where Everybody Knows Your (Screen) Name: Online Games as 'Third Places,"' J. Comput. Commun., vol. 11, no. 4, pp. 885-909, Jul. 2006.

[4] G. Yoon and P. T. Vargas, "Know Thy Avatar: The Unintended Effect of Virtual-Self Representation on Behavior," Psychol. Sci., vol. 25, no. 4, pp. 1043-1045, 2014.

[5] P. W. Ballantine and B. A. S. Martin, "Forming parasocial relationships in online communities," ACR North Am. Adv., 2005.

[6] A. Vasalou and A. N. Joinson, "Me, myself and I: The role of interactional context on selfpresentation through avatars," Comput. Human Behav., vol. 25, no. 2, pp. 510-520, Mar. 2009.

[7] D. Williams, T. L. M. Kennedy, and R. J. Moore, "Behind the avatar: The patterns, practices, and functions of role playing in MMOs," Games Cult., vol. 6, no. 2, pp. 171200, Mar. 2011.

[8] G. Hofstede and M. H. Bond, "Hofstede's Culture Dimensions," J. Cross. Cult. Psychol., vol. 15, no. 4, pp. 417-433, Dec. 1984.

[9] F. T. Mcandrew and C. R. dejonge, "Electronic person perception: What do we infer about people from the style of their email messages?," Soc. Psychol. Personal. Sci., vol. 2, no. 4, pp. 403-407, 2011.

[10] J. B. Walther, T. Loh, and L. Granka, "Let me count the ways the interchange of verbal and nonverbal cues in computer-mediated and face-to-face affinity," Journal of Language and Social Psychology, vol. 24, no. 1. pp. 3665, Mar-2005.

[11] K. M. Cornetto and K. L. Nowak, "Utilizing usernames for sex categorization in computer- mediated communication: Examining perceptions and accuracy," Cyberpsychology Behav., vol. 9, no. 4, pp. 377-387, Aug. 2006.

[12] S. V. Rouse and H. A. Haas, "Exploring the accuracies and inaccuracies of personality perception following internet-mediated communication," J. Res. Pers., vol. 37, no. 5, pp. 446-467, 2003.

[13] M. D. Back, S. C. Schmukle, and B. Egloff, "How extraverted is honey.bunny77@hotmail.de? Inferring personality from e-mail addresses," J. Res. Pers., vol. 42, no. 4, pp. 1116-1122, Aug. 2008.

[14] B. M. Okdie, R. E. Guadagno, F. J. Bernieri, A. L. Geers, and A. R. McLarney-Vesotski, "Getting to know you: Face-to-face versus online interactions," Comput. Human Behav., vol. 27, no. 1, pp. 153-159, Jan. 2011.

[15] M. D. Back et al., "Facebook profiles reflect actual personality, not self-idealization," Psychol. Sci., vol. 21, no. 3, pp. 372-374, 2010.

[16] J. M. Stopfer, B. Egloff, S. Nestler, and M. D. Back, "Being popular in online social networks: How agentic, communal, and creativity traits relate to judgments of status and liking," J. Res. Pers., vol. 47, no. 5, pp. 592-598, 2013.

[17] S. Vazire and S. D. Gosling, "e-Perceptions: Personality Impressions Based on Personal Websites.," J. Pers. Soc. Psychol., vol. 87, no. 1, pp. 123-132, 2004.

[18] M. Holzwarth, C. Janiszewski, and M. M. Neumann, "The influence of avatars on online consumer shopping behavior," J. Mark., vol. 70, no. 4, pp. 19-36, Oct. 2006.

[19] L. D. Taylor, "Avatars and emotional engagement in asynchronous online communication," Cyberpsychology, Behav. Soc. Netw., vol. 14, no. 4, pp. 207-212, Apr. 2011.

[20] C. Nass and Y. Moon, "Machines and Mindlessness: Social Responses to Computers," 2000.

[21] L. P. Naumann, S. Vazire, P. J. Rentfrow, and S. D. Gosling, "Personality judgments based on physical appearance," Personal. Soc. Psychol. Bull., vol. 35, no. 12, pp. 1661-1671, Dec. 2009.

[22] S. Wohlrab, B. Fink, P. M. Kappeler, and G. Brewer, "Differences in Personality Attributions Toward Tattooed and Nontattooed Virtual Human Characters," J. Individ. Differ., vol. 30, no. 1, pp. 1-5, 2009.

[23] K. L. Nowak and C. Rauh, "Choose your 'buddy icon' carefully: The influence of avatar 
androgyny, anthropomorphism and credibility in online interactions," Comput. Human Behav., vol. 24, no. 4, pp. 1473-1493, Jul. 2008.

[24] K. L. Nowak, M. A. Hamilton, and C. C. Hammond, "The effect of image features on judgments of homophily, credibility, and intention to use as avatars in future interactions," Media Psychol., vol. 12, no. 1, pp. 50-76, Jan. 2009.

[25] Y. B. Kafai, D. A. Fields, and M. S. Cook, "Your second selves: Player-designed avatars," Games Cult., vol. 5, no. 1, pp. 23-42, Jan. 2010.

[26] R. A. Dunn and R. E. Guadagno, "My avatar and me - Gender and personality predictors of avatar-self discrepancy," Comput. Human Behav., vol. 28, no. 1, pp. 97-106, Jan. 2012.

[27] A. Vasalou, A. Joinson, T. Bänziger, P. Goldie, and J. Pitt, "Avatars in social media: Balancing accuracy, playfulness and embodied messages," vol. 66, no. 11, pp. 801811, Nov. 2008.

[28] A. E. Park and T. B. Henley, "Personality and Fantasy Game Character Preferences," Imagin. Cogn. Pers., vol. 27, no. 1, pp. 37-46, Sep. 2007.

[29] O. Gillath, A. J. Bahns, F. Ge, and C. S. Crandall, "Shoes as a source of first impressions," J. Res. Pers., vol. 46, no. 4, pp. 423-430, Aug. 2012.

[30] P. Borkenau and A. Liebler, "Trait Inferences: Sources of Validity at Zero Acquaintance," $J$. Pers. Soc. Psychol., vol. 62, no. 4, pp. 645657, 1992.

[31] K. L. Nowak and C. Rauh, "The influence of the avatar on online perceptions of anthropomorphism, androgyny, credibility, homophily, and attraction," J. Comput. Commun., vol. 11, no. 1, 2005.

[32] Z. Ruttkay, "Cultural dialects of real and synthetic emotional facial expressions," in $A I$ and Society, 2009, vol. 24, no. 3, pp. 307-315.

[33] M. Rehm, Y. Nakano, E. André, and T. Nishida, "Culture-specific first meeting encounters between virtual agents," in International Workshop on Intelligent Virtual Agents, 2008, pp. 223-236.

[34] C. Bartneck, T. Takahashi, and Y. Katagiri, "Cross-Cultural Study of Expressive Avatars," in Proceedings of the Social Intelligence Design 2004, 2004, pp. 21-28.

[35] T. Koda, "Interpretation of Emotionally Expressive Characters in an Intercultural Communication," 2004, pp. 862-868.

[36] G. Hofstede and R. R. McCrae, "Personality and Culture Revisited: Linking Traits and
Dimensions of Culture," Cross-Cultural Res., vol. 38, no. 1, pp. 52-88, Feb. 2004.

[37] D. Oyserman, H. M. Coon, and M. Kemmelmeier, "Rethinking Individualism and Collectivism: Evaluation of Theoretical Assumptions and Meta-Analyses," 2002.

[38] R. Benedict, Patterns of culture. Houghton Mifflin, 2005.

[39] G. H. Hofstede, "Culture's Consequences, Second Edition: Comparing Values, Behaviors, Institutions and Organizations Across Nations," in Edn, Sage Publications, Inc, Thousand Oaks, 2001.

[40] Hofstede Insights, "The 6 dimensions of national culture," Hofstede Insights, 2018.

[41] A. J. Watson and E. Brunswik, "Perception and the Representative Design of Psychological Experiments.," Philos. Q., 1958.

[42] G. Hofstede, "Hofstede insights," 2019. [Online]. Available: https://www.hofstedeinsights.com/country-

comparison/greece,pakistan,russia,singapore/.

[43] O. P. John, E. M. Donahue, and K. R. L, The Big Five Inventory--Versions 4a and 54. 1991.

[44] O. P. John and S. Srivastava, "The Big Five trait taxonomy: History, measurement, and theoretical perspectives," Handb. Personal. Theory Res., 1999.

[45] B. Rammstedt and O. P. John, "Measuring personality in one minute or less: A 10-item short version of the Big Five Inventory in English and German," J. Res. Pers., vol. 41, no. 1, pp. 203-212, Feb. 2007.

[46] D. C. Funder and C. D. Sneed, "Behavioral Manifestations of Personality: An Ecological Approach to Judgmental Accuracy," J. Pers. Soc. Psychol., vol. 64, no. 3, pp. 479-490, 1993. 